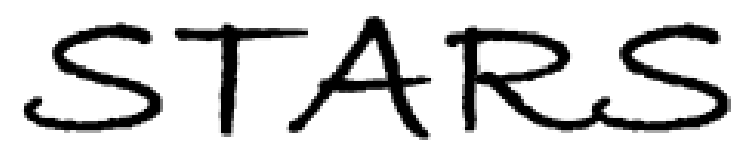

University of Central Florida

STARS

Faculty Bibliography 1990s

Faculty Bibliography

$1-1-1998$

\title{
Singular structure in 4D simplicial gravity
}

\author{
S. Catterall \\ R. Renken \\ University of Central Florida \\ J. Kogut
}

Find similar works at: https://stars.library.ucf.edu/facultybib1990

University of Central Florida Libraries http://library.ucf.edu

This Article is brought to you for free and open access by the Faculty Bibliography at STARS. It has been accepted for inclusion in Faculty Bibliography 1990s by an authorized administrator of STARS. For more information, please contact STARS@ucf.edu.

\section{Recommended Citation}

Catterall, S.; Renken, R.; and Kogut, J., "Singular structure in 4D simplicial gravity" (1998). Faculty Bibliography 1990s. 2197.

https://stars.library.ucf.edu/facultybib1990/2197

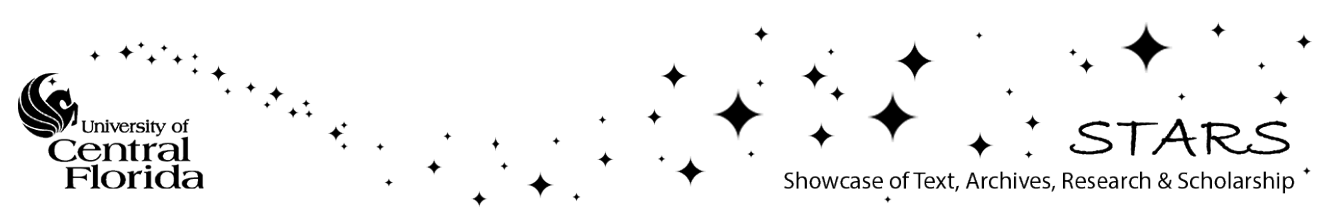




\title{
Singular structure in 4D simplicial gravity
}

\author{
S. Catterall ${ }^{\mathrm{a}}$, R. Renken ${ }^{\mathrm{b}}$, J. Kogut ${ }^{\mathrm{c}}$ \\ a Physics Department, Syracuse University, Syracuse, NY 13244, USA \\ b Physics Department, University of Central Florida, Orlando, FL 32816, USA \\ ${ }^{\mathrm{c}}$ Loomis Laboratory, University of Illinois, 1110 W. Green St., Urbana, IL 61801, USA
}

Received 11 September 1997

Editor: H. Georgi

\begin{abstract}
We show that the phase transition previously observed in dynamical triangulation models of quantum gravity can be understood as being due to the creation of a singular link. The transition between singular and non-singular geometries as the gravitational coupling is varied appears to be first order. (C) 1998 Elsevier Science B.V.
\end{abstract}

Dynamical triangulations (DT) furnish a powerful approach to the problem of defining and studying a non-perturbative theory of quantum gravity. Simply put, the functional integral over (euclidean) fourgeometries is defined as some scaling limit of a sum over abstract simplicial manifolds. In two dimensions this approach has been very successful [1]. In addition to the calculation of gravitationally dressed anomalous dimensions, the DT approach, being discrete, allows for the use of non-perturbative methods such as computer simulation. In two dimensions this has yielded new results; the measurement of fractal dimensions characterizing the quantum geometry [2], power law behavior of matter field correlators on geodesic paths [3] and insight into the problem of formulating a renormalization group for quantum gravity [4].

The observation of a two phase structure for the four dimensional models was noticed early on [5]. This led to the speculation that a continuum theory of gravity could be constructed by taking an appropriate scaling limit near the phase transition. How- ever, in [6], evidence was presented that the transition was discontinuous. Such a scenario would rule out arguably the simplest possibility - that a continuum theory could be obtained by tuning the theory to some critical (gravitational) coupling.

Independent of these issues it was noted in [7] that the crumpled phase of this model possessed a singular structure - that the sum over triangulations was dominated by those with a single link common to a very large number of four-simplices. The endpoints of this link, termed singular vertices, were shared by a number of four-simplices which diverged linearly with the total triangulation volume ${ }^{1}$.

In this note we have studied how this singular structure changes as the gravitational coupling is varied. We find evidence that the transition is driven by the creation of singular vertices as the coupling is lowered, singular vertices being absent in the

\footnotetext{
${ }^{1}$ Singular vertices were first noticed in [8].
} 
branched polymer (large coupling) phase. We support our observations by appealing to a mean-field argument due to Bialas at al [9].

The model we have investigated is defined by the grand canonical partition function

$Z_{\mathrm{GC}}=\sum_{T} e^{-\kappa_{4} N_{4}+\kappa_{0} N_{0}}$

The coupling $\kappa_{0}$ plays the role of an (inverse) bare gravitational constant conjugate to a discrete analog of the integrated curvature - the number of vertices $N_{0}$. In practice we use $\kappa_{4}$ (the bare cosmological constant) to fix the volume (number of foursimplices $N_{4}$ ) and consider a canonical partition function

$Z_{C}\left(\kappa_{0}, V\right)=\sum_{T_{V}} e^{\kappa_{0} N_{0}}$

The class of triangulations $T_{V}$ with volume $V$ is further restricted to be those with the global topology of the four-sphere together with a local manifold constraint - that the vicinity of any point should be homeomorphic to a four-ball.

We use a Monte Carlo algorithm, described in [10], to sample the dominant contributions to $Z_{C}$.
Fig. 1 shows a plot of the local volume of the most singular vertex $\left\langle\omega_{0}\right\rangle$ (i.e. the number of simplices sharing that vertex) against coupling $\kappa_{0}$ for lattices with volume $8 K, 16 K$ and $32 K$.

Deep in the crumpled phase it is clear that $\left\langle\omega_{0}\right\rangle$ scales linearly with system size as expected. For sufficiently large coupling $\kappa_{0}$ it is small, the ratio $\left\langle\omega_{0}\right\rangle / V$ approaching zero as $V \rightarrow \infty$. The behavior of $\omega_{0}$ for intermediate coupling is somewhat complex - on the $8 K$ lattice it falls rather slowly for $\kappa_{0}<1$, kinks for $\kappa_{0} \sim 1$, falls more rapidly until $\kappa_{0} \sim 1.8$ and then plateaus until $\kappa_{0} \sim 2$. We will call the regime from $\kappa_{0} \sim 1$ to $\kappa_{0} \sim 2$ the mixed region. For couplings larger than this $\left\langle\omega_{0}\right\rangle$ rapidly approaches its asymptotic value. A similar picture is seen at $16 K$ - the volume $\omega_{0}$ falls slowly up to $\kappa_{0} \sim 2$ after which it falls quickly to a small plateau for $\kappa_{0} \sim 2$ 2.2. For couplings larger than this the value of $\omega_{0}$ quickly approaches its limiting value. Thus the behavior is similar to the $8 K$ data except that the mixed region is now much smaller. The arrow on the plot indicates the approximate position of the usual phase transition as revealed by measurements of the vertex susceptibility $\frac{1}{V}\left(\left\langle N_{0}^{2}\right\rangle-\left\langle N_{0}\right\rangle^{2}\right)$ for large vol-

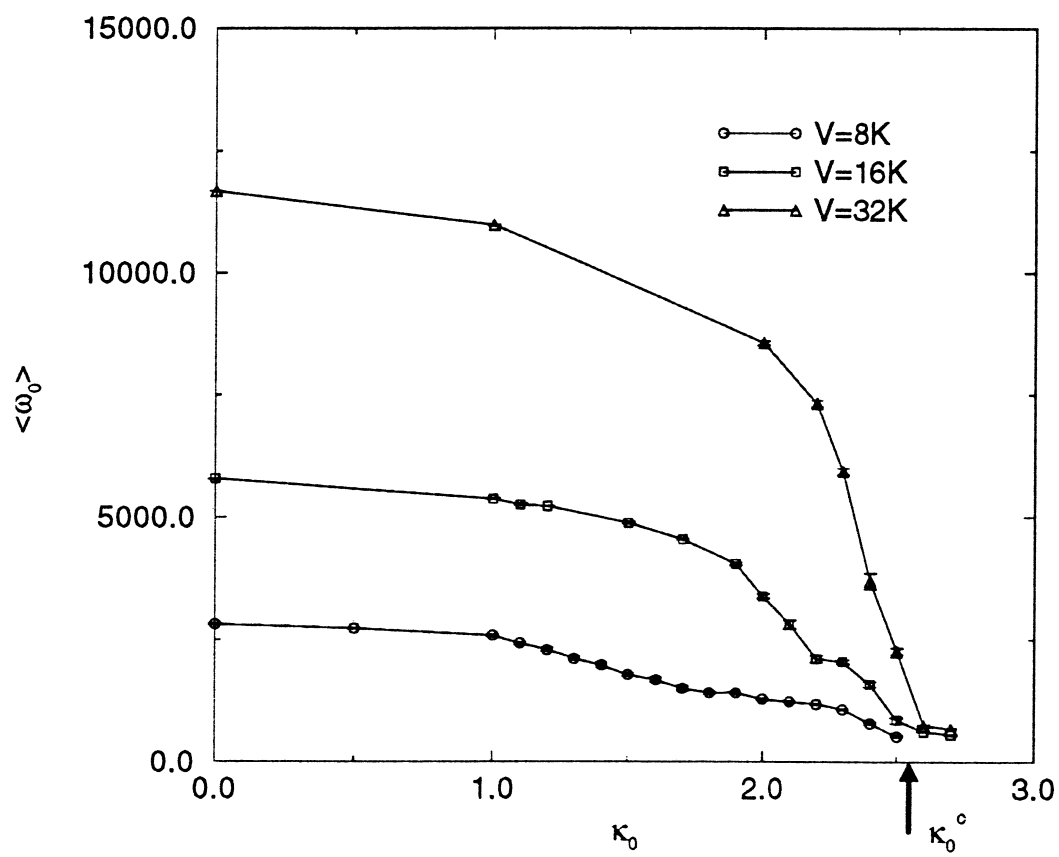

Fig. 1. Singular vertex volume vs $\kappa_{0}$ 


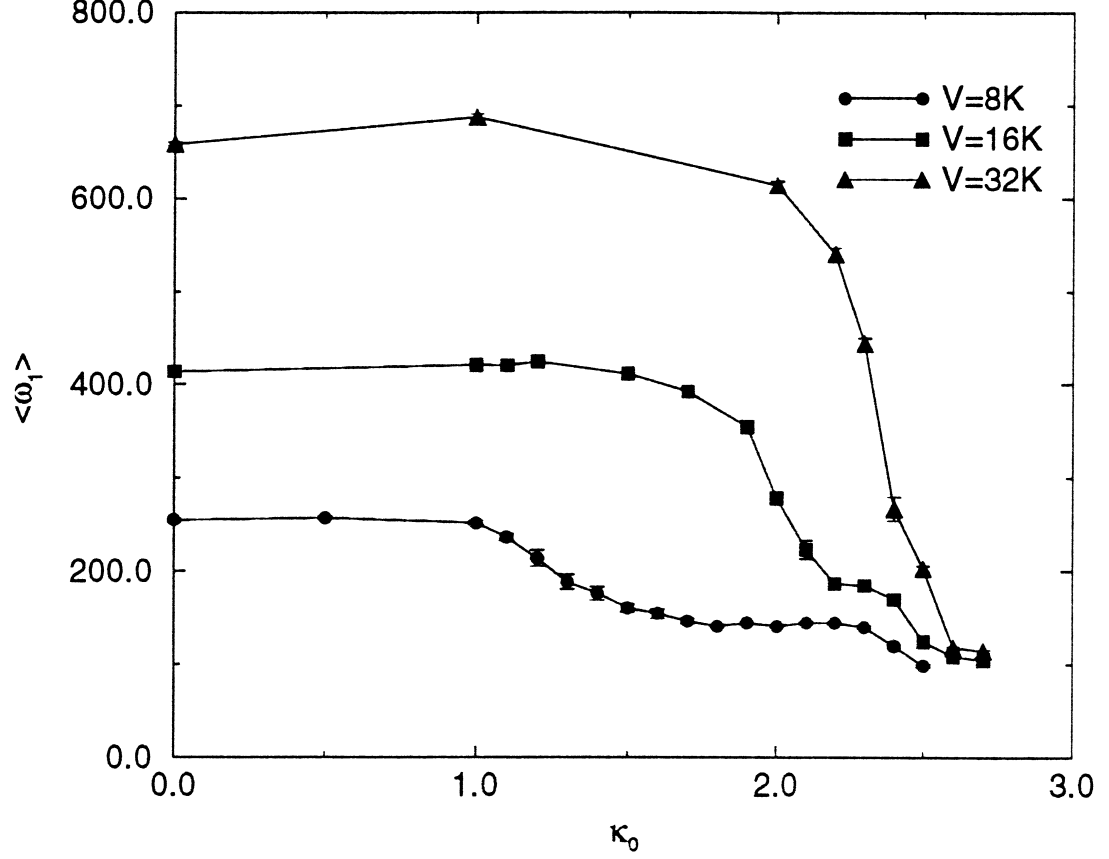

Fig. 3. Singular link volume vs $\kappa_{0}$

the peak in $\chi_{0}$ with volume, characteristic of a first order phase transition.

If we run simulations close to $\kappa_{0}^{c}$ for large vol- umes we see signs of meta-stability - the system tunnels back and forth between two distinct states one is branched polymer-like and contains no singu-

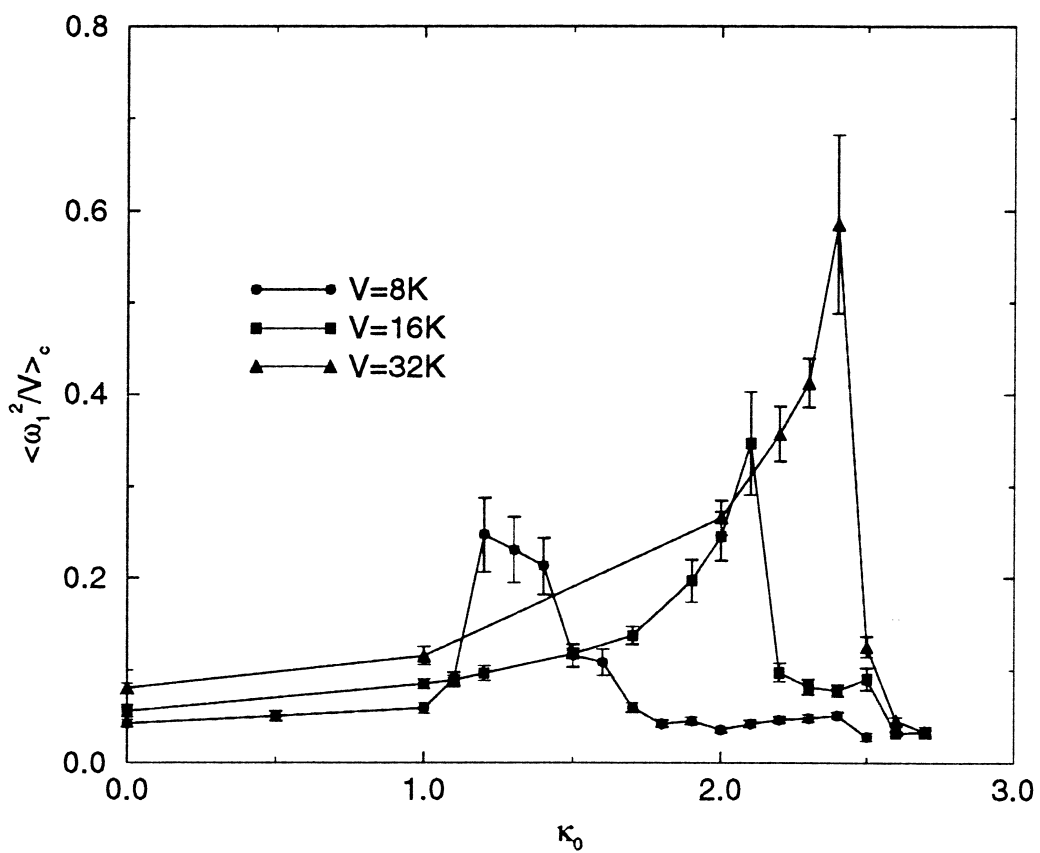

Fig. 4. Fluctuations in singular link volume $\chi_{1}$ 


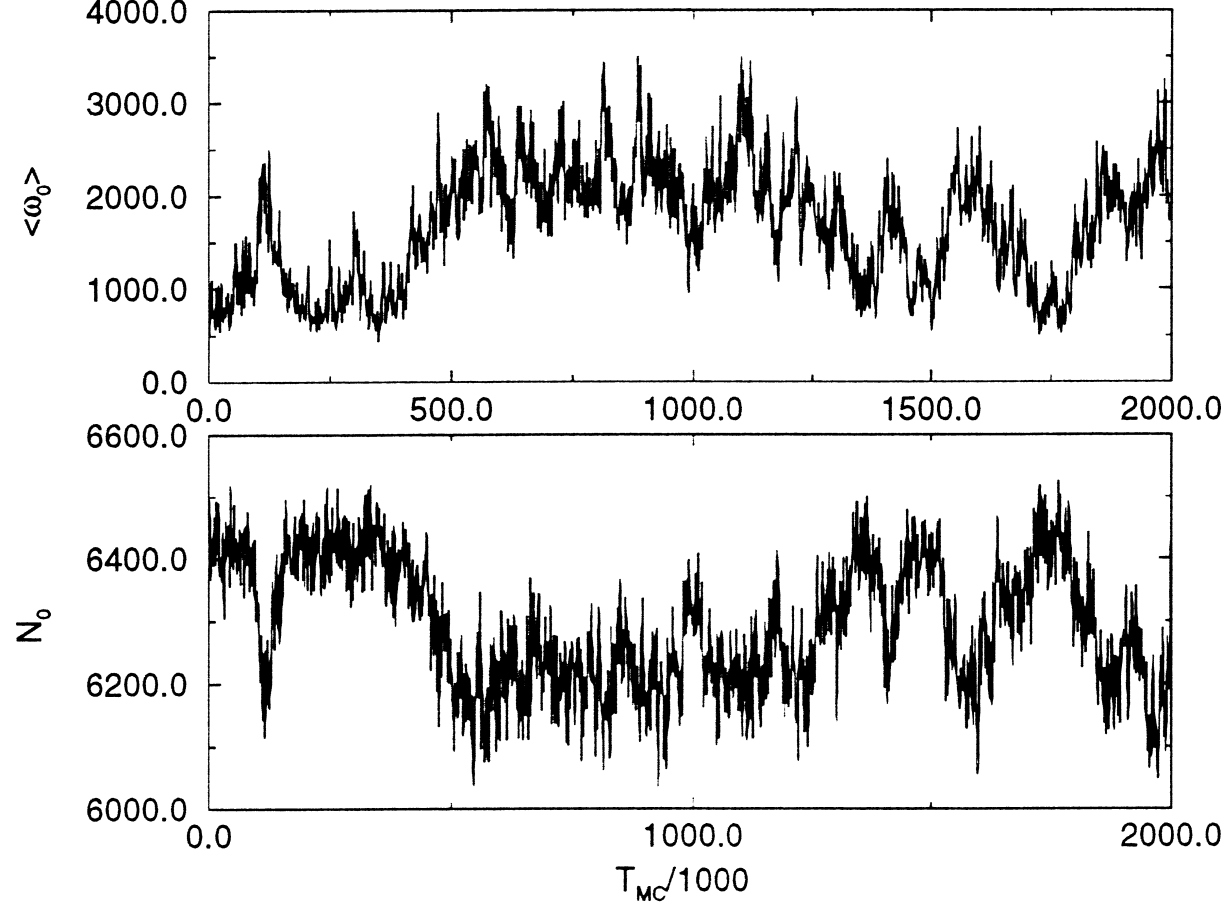

Fig. 5. Correlation of vertex number $N_{0}$ with singular vertex volume

lar vertex and the other contains one or more remnant singular vertices. This type of behavior has been seen before and is a strong indicator of a discontinuous phase transition.

The correlation between the action $N_{0}$ and singular vertex volume $\omega_{0}$ can be seen easily in Fig. 5 where time series of the two quantities are plotted. There is clearly a perfect (anti)correlation - fluctuations to larger vertex numbers are accompanied by precisely matching decreases in the singular vertex volume. This is yet another indicator that the physics of the phase transition corresponds exactly to the fluctuations in the singular structure.

It is possible to gain further insight into the nature of this transition by making a couple of assumptions. Consider the microcanonical partition function $Z_{\mathrm{MC}}\left(V, N_{0}\right)$ gotten by fixing the number of vertices. Following [7] let us define a local entropy $s_{i}$ associated with a vertex $i$. This is just the number of ways of gluing together the simplices in its local volume element - hence it is a function of that local volume $s_{i}=s_{i}\left(\omega_{0}(i)\right)$. We will first assume that $Z_{\mathrm{MC}}$ can be approximated by enumerating all possible ways in which these local entropies can be assigned ${ }^{3}$. This is a drastic assumption but we are encouraged by the results of our simulations which support the notion that the transition is driven by fluctuations in the singular vertex structure. Secondly we will assume that for sufficiently small vertex density $N_{0} / V$ a mean field approximation can be used in which the local entropies are treated as independent. Thus we are led to an ansatz for the partition function of the form

$$
\begin{aligned}
Z_{\mathrm{MC}}\left(V, N_{0}\right)= & \sum_{\left\{\omega_{0}(i)\right\}} \prod_{i}^{N_{0}} s_{i}\left(\omega_{i}(i)\right) \\
& \times \delta\left(\sum_{i} \omega_{0}(i)-c V\right)
\end{aligned}
$$

The final delta function reflects the fact that at

\footnotetext{
${ }^{3}$ In the light of the results derived in [11], we should probably regard this as an effective theory valid for understanding some of the properties of the phase transition - it seems that the leading behavior of the partition function can be derived from a partitioning of simplices amongst the (non-singular) $(d-2)$-simplices the effect of the singular structure being sub-leading.
} 
least one global constraint restricts the local volumes $\omega_{0}(i)$ - that the sum of these volumes is just proportional to the volume $V$. ( $c$ the constant of proportionality is equal to five). As argued in [7] for $s_{i}\left(\omega_{0}(i)\right)$ we should take the number of triangulations of the three sphere bounding the vertex $i$. The precise functional form of $s_{i}$ is unknown but previous numerical simulations [12] and an analytic calculation of the exponential bound [13] lead to the form

$s_{i}(n) \sim \exp \left(-a n^{\sigma}\right) e^{b n}$

The constant $\sigma \sim 0.6-7$. Using the results of [9] one can write the partition function of this model in the thermodynamic limit as

$Z_{\mathrm{MC}}=\frac{1}{2 \pi} \exp \left((-\log \lambda+b) c V+\ln F(\lambda) N_{0}\right)$

where the parameter $\lambda$ is the solution of the equation

$\rho=\frac{\lambda F^{\prime}(\lambda)}{F(\lambda)}$

and

$F(\lambda)=\sum_{\omega} s^{\prime}(\omega) \lambda^{\omega}$

The prime indicates that the leading exponential piece in $s_{i}$ has been trivially factored out (it can be seen in the leading term in Eq. (5)) and so $F$ depends only on the sub-exponential pieces in $s_{i}$.

The model exhibits a two phase structure; for densities $\rho=c V / N_{0}$ with $\rho>\rho_{c}=\frac{F^{\prime}(1)}{F(1)}$ the parameter $\lambda=1$ and the system is in a collapsed phase where vertices of small order behave independently and the global constraint is satisfied by a small number of vertices which are shared by a number of simplexes on the order of the volume. This regime is identified with the crumpled phase of the dynamical triangulation model. Conversely for $\rho<\rho_{c}$ the parameter $\lambda$ varies between zero and one and the free energy varies continuously with $\rho$. The distribution of vertex orders then behaves as

$p(n) \sim \omega(n) e^{-n \log \lambda}$

This phase is then identified with the branched polymer phase in the dynamical triangulation model. As $\rho$ (and hence $N_{0}$ ) is varied (by varying the coupling $\kappa_{0}$ ) the system moves between these two phases. This phase structure is seen for any value of the parameter $\sigma<1$. Indeed any sub-exponential or power law behavior will suffice.

Our simulations are run in the canonical ensemble where a sum over vertices is taken

$Z_{C}=\sum_{N_{0}} Z_{\mathrm{MC}} \exp \kappa_{0} N_{0}$

If we use the mean-field approximation for $Z_{\mathrm{MC}}$ this integration can be done [14]. The model again exhibits a two phase structure with a first order phase transition separating the crumpled phase from the branched polymer phase. This then is in agreement with the results of our simulations.

In our mean field arguments we have only considered the vertex sector of the model - clearly the links can be considered as possessing a local entropy too. In this case this entropy would be equal to the number of triangulations of the two-sphere dual to the link. Similar arguments would then lead to the prediction of a two phase structure for the links - a collapsed phase with singular links and a fluid phase in which the link distribution behaves exponentially with link local volume. The coupling $\kappa_{0}$ at which this transition occurred would seemingly be independent of the coupling at which singular vertices appear. Although our simulations are consistent with this scenario at small volume it appears that these two transition points merge in the infinite volume limit. Of course, the vertex and link sectors are not truly independent and it is presumably the presence of these residual constraints that is responsible for the coincidence of the two transitions.

In summary, we have reported numerical results which strongly support the idea that the phase transition observed in $4 \mathrm{~d}$ simplicial gravity is associated with the creation of singular geometries. These singular structures, composed of a single singular link between two singular vertices, dominate at strong coupling. As the coupling $\frac{1}{\kappa_{0}}$ is decreased they eventually disappear. This process appears to be discontinuous and is the origin of the first order nature of the transition. Simple mean field arguments support these conclusions.

This work was supported in part by NSF Grant PHY-9503371 and DOE Grant DE-FG0285ER40237. We acknowledge useful conversations with B. Petersson. 


\section{References}

[1] F. David, "Simplicial Quantum Gravity and Random Lattices," (hep-th/9303127), Lectures given at Les Houches Summer School on Gravitation and Quantizations, Session LVII, Les Houches, France, 1992,

J. Ambjørn, “Quantization of Geometry.'” (hep-th/9411179), Lectures given at Les Houches Summer School on Fluctuating Geometries in Statistical Mechanics and Field Theory, Session LXII, Les Houches, France, 1994,

P. Ginsparg and G. Moore, "Lectures on 2D Gravity and 2D String Theory,', (hep-th/9304011), Lectures given at TASI Summer School, Boulder, CO, 1992,

P. Di Francesco, P. Ginsparg and J. Zinn-Justin, "2-d Gravity and Random Matrices,', (hep-th/9306153), Phys. Rep. 254 (1995) 1.

[2] S. Catterall, G. Thorleifsson, M. Bowick and V. John, Phys. Lett. B 354 (1995) 58.

J. Ambjørn, J. Jurkiewicz and Y. Watabiki, Nucl. Phys. B 454 (1995) 313.

[3] J. Ambjorn and K. Anagnostopoulos, Phys. Lett. B 388 (1996) 713.

[4] G. Thorleifsson and S. Catterall, Nucl. Phys. B 461 (1996) 350. R. Renken, Phys. Rev. D 50 (1994) 5130.

[5] M. Agishtein and A. Migdal, Nucl. Phys. B 385 (1992) 395. J. Ambjørn and J. Jurkiewicz, Phys. Lett. B 278 (1992) 42. 1908.

S. Catterall, J. Kogut and R. Renken, Phys. Lett. B 328 (1994) 277.

B. de Bakker and J. Smit, Nucl. Phys. B 437 (1995) 239.

[6] P. Bialas, Z. Burda, A. Krzywicki and B. Petersson Nucl. Phys. B 472 (1996) 293.

B. de Bakker, Phys. Lett. B 389 (1996) 238.

[7] S. Catterall, G. Thorleifsson, J. Kogut and R. Renken, Nucl. Phys. B 468 (1996).

[8] T. Hotta, T. Izubuchi and J. Nishimura, Prog. Theor. Phys. 94 (1995) 263.

[9] P. Bialas, D. Johnston and Z. Burda, Nucl. Phys. B 493 (1997) 505.

P. Bialas, Z. Burda, B. Petersson and J. Tabaczek, Nucl. Phys. B 495 (1997) 463.

[10] S. Catterall, Computer Physics Communications 87 (1995) 409.

[11] J, Tabaczek: Diplomarbeit Bielefeld Juni 1997.

[12] S. Catterall, R. Renken and J. Kogut, Phys. Lett. B 342 (1995) 53.

J. Ambjorn and S. Varsted, Phys. Lett. B 226 (1991) 285.

[13] “The Geometry of Dynamical Triangulations", J. Ambjorn, M. Carfora and A. Marzuoli. (Springer Verlag), to be published.

[14] P. Bialas and Z. Burda, hep-lat/9707028. 